\title{
CARGAS E DESGASTES DE TRABALHO VIVENCIADOS ENTRE TRABALHADORES DE SAÚDE EM UM HOSPITAL DE ENSINO
}

Leni de Lima SANTANA a , Fernanda Moura D’Almeida MIRANDA ${ }^{\text {, }}$ Márcia Eiko KARINOc, Patrícia Campos Pavan BAPTISTA ${ }^{\text {, }}$ Vanda Elisa Andres FELLI ${ }^{e}$, Leila Maria Mansano SARQUIS

\section{RESUMO}

Estudo exploratório, de caráter descritivo e de abordagem quantitativa, alicerçado nas categorias "processo de trabalho”, "cargas de trabalho" e "desgaste” em um hospital de ensino em Curitiba, região sul do Brasil. Neste artigo, são caracterizadas as cargas e os desgastes vivenciados em um hospital universitário, captados pelo estudo prévio intitulado "Sistema de monitoramento da saúde do trabalhador de enfermagem" (SIMOSTE). Os resultados demonstram que o gênero feminino foi o mais acometido (85,9\%); os profissionais mais afetados foram os auxiliares de enfermagem $(53,1 \%)$. O maior número de afastamentos ocorreu por doenças do sistema osteoarticular (25,2\%) e, dentre as cargas apresentadas, as mais expressivas foram as mecânicas e fisiológicas, com 33,06\%, cada. Estes resultados poderão subsidiar estratégias de intervenção nas políticas direcionadas à saúde do trabalhador, para assegurar uma melhor qualidade de vida a este profissional e, por consequência, promover melhorias na qualidade da assistência prestada ao usuário.

Descritores: Enfermagem. Saúde do trabalhador. Carga de trabalho. Processo saúde-doença. Pessoal de saúde.

\section{RESUMEN}

Estudio exploratorio, descriptivo y cuantitativo, basado en el proceso de trabajo, las cargas de trabajo y el desgaste en un hospital universitario en Curitiba, sur de Brasil. En este artículo se caracteriza la carga y el estrés experimentado en un hospital universitario, planteada por un estudio previo titulado "Sistema de vigilancia de la salud de los trabajadores de enfermería". Los resultados muestran que las mujeres (85,9\%) y los auxiliares de enfermería eran los profesionales más afectados (53,1\%). El mayor número de ausencia del trabajo se debieron a enfermedades del sistema musculoesquelético (25,2\%) y entre los cargos presentados, los más significativos fueron el mecánico y el fisiológico con el 33,06\% cada uno. Estos resultados pueden apoyar las estrategias de intervención en las políticas dirigidas a la salud de los trabajadores, para garantizar una mejor calidad de vida a este trabajo y promover mejoras en la calidad de la atención prestada al usuario.

Descriptores: Enfermería. Salud laboral. Carga de trabajo. Proceso salud-enfermedad. Personal de salud.

Título: Descripción de cargas de trabajo y el desgaste experimentado entre trabajadores de salud en un hospital universitario.

\section{ABSTRACT}

This is an exploratory, descriptive and quantitative study, based on the following categories: work process, workloads and fatigue in a teaching hospital in Curitiba in the southern region of Brazil. The article characterizes the load and stress experienced in a university hospital, based on a previous study entitled "System for monitoring the health of nursing workers" (SIMOSTE). The results show that females were the most affected (85.9\%) and the most affected professionals were nursing assistants (53.1\%). The highest number of sick leaves was due to diseases of the osteoarticular system (25.2\%) and the most significant loads were mechanical and physiological with 33.06\% each. These results may support intervention strategies in the policies directed toward the workers' health to ensure a better quality of life and consequently improve the quality of care provided to the user.

Descriptors: Nursing. Occupational health. Workload. Health-disease process. Health personnel. Title: Description of workloads and fatigue experienced among health workers in a teaching hospital.

a Mestranda pelo Programa de Pós-Graduação em Enfermagem da Universidade Federal do Paraná - UFPR, Curitiba, Paraná, Brasil.

b Doutoranda pelo Programa de Pós-Graduação em Enfermagem da UFPR, Curitiba, Paraná, Brasil.

c Doutora, Docente da Universidade Estadual de Londrina (UEL), Londrina, Paraná, Brasil.

d Doutora, Docente da Escola de Enfermagem da Universidade de São Paulo - EEUSP, Ribeirão Preto, São Paulo, Brasil.

e Doutora, Docente da EEUSP, Ribeirão Preto, São Paulo, Brasil

f Doutora, Docente do Programa de Pós-Graduação da UFPR, Curitiba, Paraná, Brasil. 


\section{INTRODUÇÃO}

As pesquisas desenvolvidas na área de saúde do trabalhador, sob diferentes referenciais teórico-metodológicos, apontam que os trabalhadores de enfermagem sofrem uma série de problemas de saúde relacionados com o trabalho(1).

Na concepção histórico-social, dada à inserção desses trabalhadores na prestação de serviços de saúde, apreende-se que os mesmos interagem com uma variedade de cargas no seu processo de trabalho as quais são geradoras de processos de desgaste, conferindo-lhes perfil de morbidade característico ${ }^{(2-5)}$.

Diante desta realidade, é necessário diagnosticar a exposição às cargas de trabalho que ocorre na prática destes profissionais em diferentes realidades, para tornar possível uma proposta de monitoramento a ser implementada em diferentes cenários, com a finalidade de subsidiar intervenções que possam reduzir essa exposição e os desgastes por ela gerados.

Neste estudo, tornou-se possível a captação das cargas de trabalho e desgaste ocorridos com os trabalhadores de enfermagem e outros trabalhadores de saúde por meio do Sistema de Monitoramento da Saúde do Trabalhador de Enfermagem (SIMOSTE) ${ }^{(6)}$ o qual consiste em um sistema de informação elaborado a partir de um projeto de pesquisa em desenvolvimento no cenário nacional. O mesmo foi implementado com apoio técnico da informática e assessoria da Escola de Enfermagem da Universidade de São Paulo (EEUSP), com financiamento da Fundação de Apoio à Pesquisa do estado de São Paulo (FAPESP).

Este sistema tem como objetivo captar os agravos à saúde dos trabalhadores de enfermagem e de outros trabalhadores de saúde bem como dos seus determinantes, potenciais geradores de desgaste e fortalecimento. O banco é alimentado nas instituições participantes do projeto com informações obtidas dos Comunicados de Acidentes de Trabalho (CAT) e dos afastamentos decorrentes de atestados médicos.

Os dados obtidos nas unidades regionais são encaminhados ao coordenador nacional do projeto, tratados e devolvidos para as instituições com sugestões de adoção de medidas preventivas. Implantado desde 2008, os dados são armazenados e trabalhados estatisticamente ${ }^{(7)}$.

A partir das análises realizadas são propostas intervenções nos ambientes laborais visando à melhoria nas condições de trabalho. Considerando os dados captados nos primeiros meses de sua implantação, obtivemos um diagnóstico situacional preliminar das cargas de trabalho e dos processos de desgaste gerados nos trabalhadores de saúde.

Assim, o presente estudo tem por objetivo caracterizar os trabalhadores de saúde, as cargas e os desgastes de trabalho em um hospital universitário no sul do Brasil.

\section{MÉTODO}

Trata-se de uma pesquisa descritiva, quantitativa e retrospectiva ancorada nas categorias: cargas de trabalho e desgaste. Para a coleta de dados utilizaram-se as variáveis: sexo, carga horária, categoria profissional, vínculo empregatício, tipo de ocorrência e cargas ou desgastes referentes a um hospital situado na região sul do Brasil, denominado HT-S, contidos no banco de dados do SIMOSTE.

Foram utilizados os dados quantitativos captados no ano de 2009 e consolidados no banco nacional. A população foi constituída pela totalidade dos trabalhadores de saúde do referido cenário, hospital de ensino no sul do país, a qual é constituída de 1.490 trabalhadores, dentre os quais 407 são trabalhadores de enfermagem. A amostra captada foi de 128 trabalhadores de saúde o que corresponde à totalidade de trabalhadores de saúde que tiveram um período de afastamento no ano de 2009.

Após a aprovação do projeto pelo Comitê de Ética em Pesquisa da Escola de Enfermagem da USP, sob o protocolo $n^{\circ} 718 / 2008$, os dados foram coletados e, posteriormente, sistematizados de acordo com a estatística descritiva segundo as frequências relativas e absolutas e analisados quantitativamente.

\section{RESULTADOS}

Foram registrados 128 casos de adoecimento relacionados ao trabalho no ano de 2009 no cenário de estudo. Quanto ao gênero houve uma predominância do sexo feminino com 110 (85,9\%) dos casos. No que se refere à carga de trabalho semanal realizada pelos trabalhadores de saúde, percebeu-se que a maioria destes, ou seja, $106(82,8 \%)$ profissionais, trabalha entre 20 a 40 horas por semana no local em que se desenvolveu o estudo.

A enfermagem aparece em primeiro lugar como a categoria profissional mais atingida, com um 
total de $83(64,8 \%)$ profissionais acidentados sendo que destes, $68(53,1 \%)$ são auxiliares e $15(11,7 \%)$ técnicos de enfermagem. Percentual este bastante significativo para a profissão uma vez que, embora de categorias diferentes estes profissionais realizem procedimentos técnico-operacionais semelhantes no atendimento ao paciente.

Com relação à situação no mercado de trabalho $106(82,8 \%)$ são trabalhadores celetistas, ou seja, amparados pela Consolidação das Leis do Trabalho (CLT) e 22 (17,2\%), servidores públicos estatutários.

A maioria dos afastamentos constatados refere-se a licenças médicas, 96 casos, representando $75,0 \%$. Destes, 32 (25,2\%) foram causados principalmente por doenças do sistema osteoarticular; 27 $(21,3 \%)$ foram consequências de causas externas (traumas); 26 (20,5\%) correspondem a transtornos mentais e comportamentais; 13 (10,2\%) devido a doenças do aparelho respiratório e $07(5,5 \%)$ por doenças do aparelho circulatório, entre outras, conforme demonstrado a seguir:

No que se refere aos desgastes a que os profissionais são submetidos, os resultados demonstram que as cargas fisiológicas e mecânicas predominam com 41 (33,06\%) casos cada (Tabela 2).

\section{DISCUSSÃO}

Torna-se necessário reforçar que os dados foram captados com os trabalhadores de enfermagem e outros trabalhadores de saúde lotados na instituição onde se desenvolveu a pesquisa. O Ministério da Saúde (MS) define trabalhadores de saúde como todo profissional inserido nos serviços

Tabela 1 - Causas de afastamentos entre profissionais de saúde no HT-S segundo dados do SIMOSTE. Curitiba, PR, 2009.

\begin{tabular}{lcc}
\hline \multicolumn{1}{c}{ CID - 10* } & N & \% \\
\hline Doença do sistema osteoarticular & 32 & 25,2 \\
Consequências por causas externas (traumas) & 27 & 21,3 \\
Transtornos mentais e comportamentais & 26 & 20,5 \\
Doença do aparelho respiratório & 13 & 10,2 \\
Outras & 18 & 14,2 \\
Doença do aparelho circulatório & 07 & 5,5 \\
Sem informação & 04 & 3,1 \\
\hline Total & $\mathbf{1 2 7}$ & $\mathbf{1 0 0}$ \\
\hline
\end{tabular}

Fonte: os autores, 2012.

* Classificação Internacional de Doenças.

Tabela 2 - Cargas e desgastes a que estão expostos os trabalhadores de saúde do HT-S, segundo dados levantados no SIMOSTE. Curitiba, PR, 2009.

\begin{tabular}{lcc}
\hline \multicolumn{1}{c}{ Cargas e Desgastes } & N & \% \\
\hline Cargas fisiológicas & 41 & 33,06 \\
Cargas mecânicas & 41 & 33,06 \\
Cargas psicossociais & 24 & 19,35 \\
Cargas biológicas & 12 & 9,70 \\
Cargas físicas & 01 & 0,80 \\
Cargas químicas & 05 & 4,03 \\
\hline Total & $\mathbf{1 2 4}$ & $\mathbf{1 0 0}$ \\
\hline
\end{tabular}

Fonte: os autores, 2012 
de saúde públicos ou privados e que desempenham suas atividades laborais nestes locais ${ }^{(8)}$. Estão incluídos nessa definição todos os profissionais que desenvolvem atividades diretamente relacionadas à assistência dos pacientes e/ou clientes, assim como aqueles que pertencem aos serviços de apoio e infraestrutura para a assistência, como os de limpeza, manutenção, apoio diagnóstico e outros.

O estudo demonstra que a categoria profissional mais atingida foi a enfermagem, confirmando, assim, a grande exposição destes trabalhadores aos processos de morbidade. Apesar do número de enfermeiros ser inferior ao dos profissionais de nível médio e técnico, é possível que, devido a responsabilidade pela supervisão do trabalho de enfermagem, e pelo número reduzido de profissionais em relação às demais categorias, estes trabalhadores se afastem menos que os auxiliares e técnicos de enfermagem optando, muitas vezes, por trabalhar doentes para não deixar a equipe sem uma pessoa de referência ${ }^{(9)}$.

Também é significativo e expressivo o registro de afastamentos de trabalhadores de saúde de outras unidades de apoio como: lavanderia, limpeza e serviços de nutrição, demonstrando, assim, a exposição dos mesmos às diversas cargas de trabalho, principalmente às fisiológicas.

No cenário do estudo constata-se o alto índice de afastamentos entre as mulheres trabalhadoras, corroborando com os dados de outras pesquisas ${ }^{(10,11)}$. Muitas mulheres estão inseridas no mercado de trabalho para contribuir com a renda familiar o que pode gerar sobrecarga de atividades. O trabalho profissional pode estar relacionado a outras funções prolongando a jornada de trabalho diária em até 12 horas, o que pode contribuir com a ocorrência de acidentes pelo desgaste decorrente. Além disso, não se podem excluir as características de morbidade feminina, como distúrbios hormonais, os quais também comprometem a saúde da mulher sendo, muitas vezes responsáveis pelo absenteísmo dessa classe trabalhadora ${ }^{(9)}$.

As cargas de trabalho são elementos que interatuam dinamicamente entre si e no corpo do trabalhador, gerando processos de adaptação que se traduzem em desgaste, os quais são definidos como a perda da capacidade efetiva e/ou potencial corporal e psíquica, que engloba os processos biopsíquicos em seu conjunto ${ }^{(12)}$. Esses processos demonstram características da coletividade e definem o perfil patológico do grupo específico. O processo de desgaste muitas vezes está na produção do trabalho caracterizado pelo não registro formal das exposições vivenciadas na atividade laboral ${ }^{(10)}$.

O mundo do trabalho tem sofrido mudanças estruturais, impondo inserção diferenciada nos mercados, novas relações de trabalho, novos mecanismos de gestão, reestruturação produtiva e exigência de novos perfis profissionais. As conseqüências dessas mudanças são marcadas pela flexibilização, pela alta especialização e ao mesmo tempo a desespecialização, a polivalência (multifuncionalidade), a subcontratação, a informalidade, a perda dos direitos sociais, o desemprego e a precarização do trabalho ${ }^{(13)}$. A enfermagem enquanto prática que se insere no mundo do trabalho e na atenção à saúde, estabelece vínculos com as leis sociais e sofre o impacto dessas transformações ${ }^{(1)}$.

A exposição às cargas de trabalho pode desencadear problemas de saúde entre os trabalhadores de enfermagem ${ }^{(1)}$ e entre outros profissionais, seja, pela morbidade referida pelo profissional ou por aquela caracterizada e captada pelos exames médicos e pelos acidentes de trabalho registrados. As morbidades referidas pelos trabalhadores em estudo de monitoramento da saúde do trabalhador $^{(1)}$ foram os ferimentos perfuro-corto-contusos, as doenças infecciosas, infecto-contagiosas e parasitárias, as doenças ósteo-músculo-articulares, os problemas respiratórios, desequilíbrio mental, desgaste emocional, enxaqueca, cefaléia, problemas de pele, problemas oculares e auditivos, intoxicação por substâncias químicas e o estresse.

Entre as morbidades que não foram relatadas, porém, foram registradas após caracterização médica estão os ferimentos perfuro-corto-contusos, as doenças ósteo-músculo-articulares, os problemas urinários, os problemas respiratórios, as alterações hematológicas, as doenças infecciosas, infectocontagiosas e parasitárias, problemas gastrintestinais, enxaqueca, cefaléia, problemas ginecológicos e alterações glicêmicas. Como acidente de trabalho foi registrado apenas aqueles decorrentes de instrumentos perfurocortantes e o comprometimento com as doenças osteo-músculo-articulares ${ }^{(1)}$.

Com os problemas de saúde acima apresentados, os trabalhadores freqüentemente ausentam-se do trabalho por não suportarem as cargas a que estão expostos, por adoecerem ou acidentarem-se. Esses afastamentos evidenciam que o trabalhador sofre diversos processos de desgaste os quais geram 
danos que se manifestam comprometendo a qualidade de vida ao longo de sua existência.

A força de trabalho da enfermagem no país soma, aproximadamente, 900.000 trabalhadores ativos, distribuídos de forma muito heterogênea nas diferentes regiões, sendo que cerca de $70 \%$ dessa força de trabalho estão concentradas nas regiões sudeste e sul(14). Embora este número seja expressivo, geralmente a enfermagem trabalha em um ritmo acelerado devido a grande demanda de serviço e a escassez de trabalhadores nos ambientes de trabalho, estas condições geram afastamentos que, além de prejuízos financeiros, comprometem a assistência prestada uma vez que sobrecarrega os demais funcionários da equipe refletindo de forma negativa na qualidade do cuidado prestado ${ }^{(15)}$.

Assim, estudos realizados sobre a saúde dos trabalhadores de enfermagem ${ }^{(15,16)}$ apontam determinantes importantes no processo saúde-doença diante de tais comportamentos, porém ainda existem lacunas no conhecimento que não permitem apreendê-los na sua totalidade.

Há uma incidência maior de registros da exposição às cargas fisiológicas embora exista expressivo desgaste do trabalhador também por outras cargas (Tabela 2). O profissional da área da saúde desenvolve o trabalho muitas vezes em situação rotineira, mecânica e repetitiva, o que pode comprometer a sua saúde gerando processos de desgaste físicos os quais podem acarretar a perda ou a redução da capacidade de produzir, bem como o agravamento e aparecimento de doenças como as lesões osteoarticulares e outros agravos à saúde.

No que se referem às cargas mecânicas, estas são mais facilmente reconhecidas como acidentes de trabalho por provocarem lesões que consistem na ruptura de segmentos do corpo como, por exemplo, as contusões, fraturas, perfurações, cortes e hematomas. Também assumem importância nesse grupo, os ferimentos perfurocortantes, potencializados pelas cargas biológicas, pela capacidade em contaminar os trabalhadores com vírus importantes e letais, como o da Hepatite B e C e o vírus da Síndrome da Imunodeficiência Adquirida (AIDS). Os trabalhadores registram e buscam registrar esta exposição muitas vezes pelo medo e o comprometimento com sua saúde(10).

Para a carga psíquica, foram registrados os transtornos mentais e comportamentais que podem comprometer e agravar a saúde do trabalhador. Para esta carga o desgaste está caracterizado por supervisão estrita; ritmo acelerado; trabalho parcelado, monótono e repetitivo; dificuldade na comunicação; agressão psíquica; fadiga; tensão; estresse e insatisfação. Situações estas que podem desencadear desde acidentes de trabalho até outros agravos à saúde ${ }^{(16)}$ como, por exemplo, os distúrbios emocionais, a ansiedade e a depressão. Nas organizações de saúde é comum a diminuição de recursos humanos, sendo este o principal fator para o ritmo acelerado de trabalho.

Em estudo realizado ${ }^{(9)}$ em 2007, os autores afirmam que os trabalhadores de enfermagem estão mais expostos às cargas psíquicas, devido à insatisfação dos usuários, carência de recursos humanos e conseqüentemente à sobrecarga de trabalho.

As cargas biológicas estão presentes nos diversos serviços de saúde, desde os serviços de atenção primária até os secundários e terciários, porém, é no ambiente hospitalar que há uma maior concentração de pacientes com doenças infecciosas e infectocontagiosas e também um grande número de profissionais da saúde ${ }^{(10)}$ fator este que contribui para o aumento do risco de exposição às mesmas.

O estudo teve como cenário específico uma instituição hospitalar que é referência para atendimento de traumas e moléstias infectocontagiosas do estado do Paraná, desta forma, a concentração de pacientes com este tipo de agravo torna-se bastante expressiva aumentando, assim o risco de contaminação. A literatura ${ }^{(1)}$ tem registrado uma série de patologias decorrentes dessa exposição, como processos infecciosos localizados e sistêmicos, toxoplasmose, hepatites, síndrome da imunodeficiência adquirida, pneumonia, gripes e resfriados de diversas naturezas, infecções urinárias, entre outras.

Quanto aos registros referentes às cargas químicas, é importante ressaltar que, embora apareçam em uma pequena amostra, não significa que o trabalhador não se exponha às mesmas. A forma como o trabalho de enfermagem é organizado agrava os processos de desgaste dos trabalhadores pela exposição a este tipo de carga a qual pode ser potencializada pela interação do trabalhador com substâncias químicas em salas mal ventiladas e espaços físicos inadequados ${ }^{(17)}$. O que ocorre, é que, na maioria das vezes, os trabalhadores não apreendem esta situação como exposição e agravos à sua saúde.

As substâncias químicas são amplamente utilizadas na assistência à saúde embora poucas vezes os trabalhadores recebam informações necessárias 
sobre sua manipulação correta bem como sobre seus efeitos sobre o organismo. Apesar de algumas delas serem irritantes, tóxicas, alergênicas, carcinogênicas, teratogênicas ou mutagênicas ${ }^{(17)}$, o fato de seus efeitos nem sempre aparecem em curto prazo fazem com que os problemas decorrentes de sua exposição não sejam relatados pelos trabalhadores de saúde.

Os acidentes de trabalho ou doenças relacionadas ao trabalho continuam ocorrendo e causando prejuízos pessoais, sociais e econômicos às famílias além de elevados custos para a economia nacional, tanto de forma direta, pelos custos assistenciais e previdenciários, quanto de forma indireta, com a perda da força de trabalho e do investimento na formação do trabalhador ${ }^{(18)}$.

Os dias de afastamento decorrentes das licenças médicas e acidentes de trabalho tornam-se problema relevante considerando-se que a maioria dos serviços de saúde, entre eles os hospitalares, não prevê cobertura para os dias de falta, sobrecarregando, assim, os trabalhadores e interferindo na qualidade da assistência prestada aos usuários ${ }^{(16)}$.

\section{CONCLUSÃO}

Os resultados apresentados descrevem a situação das cargas e dos desgastes relacionados ao trabalho vivenciados pelos trabalhadores de saúde de um hospital de ensino no sul do Brasil, os quais poderão subsidiar estratégias de intervenção nas políticas direcionadas à saúde do trabalhador, para assegurar uma melhor qualidade de vida do trabalhador e por conseqüência na qualidade da assistência prestada ao usuário.

Embora as evidências e os estudos comprovem as perdas econômicas, pessoais, o comprometimento na qualidade da assistência prestada, bem como os danos físicos e psicológicos decorrentes das doenças e acidentes de trabalho, e, embora os dados apresentados neste estudo demonstrem uma alta incidência de exposição dos trabalhadores de saúde às cargas de trabalho, podemos inferir que, na realidade, estes dados estão subnotificados vista a baixa incidência de cargas físicas, químicas e biológicas.

Esta questão nos faz refletir sobre como estão, e se estão, sendo aplicadas as políticas voltadas à saúde do trabalhador nas instituições de saúde e quais estratégias necessárias frente ao papel de prevenção e controle dos agravos decorrentes da atividade profissional.
Desta forma, sugere-se que novos estudos sejam realizados a fim de diminuir as causas de adoecimento desses trabalhadores, principalmente sobre o modelo organizacional e de motivação para o trabalho.

\section{REFERÊNCIAS}

1 Felli VEA. Implantação e Avaliação do Sistema de Monitoramento da Saúde do Trabalhador de Enfermagem (SIMOSTE). São Paulo; 2010. (Relatório final apresentado ao $\mathrm{CNPq}$, para prestação de contas da Bolsa Produtividade-2, relativa ao período 2008-2011).

2 Balsamo AC, Felli VEA. Estudo sobre os acidentes de trabalho com exposição aos líquidos corporais humanos em trabalhadores da saúde de um hospital universitário. Rev Latinoam Enferm. 2006;14(3):346-353.

3 Dal Bem LW, Carvalho MB, Felli VEA. A percepção da relação sofrimento/prazer no trabalho de auxiliares de enfermagem e técnicos de enfermagem em internação domiciliária. Cogitare Enferm. 2005; (2):73-81.

4. Laurell AC, Noriega M. Processo de Produção em Saúde: trabalho e desgaste operário. São Paulo: Hucitec; 1989.

5 Felli VEA, Tronchin DMR. A Qualidade de Vida No Trabalho e a Saúde do Trabalhador de Enfermagem. In: Paulina Kurcgant, organizadora. Gerenciamento em enfermagem. Rio de Janeiro: Guanabara Koogan; 2005. p.89-107.

6 Pavan PC, Felli VEA, Miniel VA, Karino ME, Silva $\mathrm{SM}$, Tito RS, et al. A inovação tecnológica como ferramenta para monitoramento da saúde dos trabalhadores de enfermagem. Rev Esc Enferm USP. 2011;45(Esp):1621-6.

7 Felli VEA, organizadora Sistema de Monitoramento de Saúde dos Trabalhadores de Enfermagem (SIMOSTE). Manual de uso e instruções para o preenchimento do SIMOSTE. São Paulo; 2008.

8 Ministério da Saúde (BR). Portaria MTE n. 485, de 11 de novembro de 2005: dispõe sobre a Norma Regulamentadora NR 32 relativa à segurança e saúde no trabalho em serviços de saúde. Brasília (DF): MS/ Departamento de Normas Técnicas; 2005.

9 Gehring GJ, Corrêa HRF, Vieira JDN, Ferreira NA, Vieira SVR. Absenteísmo-doença entre profissionais de enfermagem da rede básica do SUS Campinas. Rev Bras Epidemiol. 2007;10(3):401-9. 
10 Miranda FMD, Júnior AVS, Petreli S, Pires MR, Soares LG, Ribeiral BN, et al. Uma contribuição à saúde dos trabalhadores: um guia sobre exposição aos fluídos biológicos. Rev Esc Enferm USP. 2011;45(4):1018-22.

11 Ribeiro EJG, Shimizu HE. Acidentes de trabalho com trabalhadores de enfermagem. Rev Bras Enferm. 2007;60(5):535-40.

12 Laurell AC, Noriega M. Processo de Produção em Saúde: trabalho e desgaste operário. São Paulo: Hucitec; 1989.

13 Felli VEA, Peduzzi M. O trabalho gerencial em enfermagem. In: Kurcgant P, coordenadora. Gerenciamento em enfermagem. Rio de Janeiro: Guanabara Koogan; 2005. cap. 1, p. 1-13.

14. Conselho Federal de Enfermagem (COFEN). Dados Estatísticos sobre a composição da força de trabalho de enfermagem [Internet]. Brasília (DF); 2006 [citado 2007 Jan 30]. Disponível em: http://www. portalcofen.com.br/
15 Sápia T, Felli VEA, Ciampone MHT. Problemas de saúde de trabalhadores de enfermagem em ambulatórios pela exposição às cargas fisiológicas. Acta Paul Enferm. 2009;22(6):808-13.

16 Costa FM, Vieira MA, Sena RR. Absenteísmo relacionado a doenças entre membros da equipe de enfermagem de um hospital escola. Rev Bras Enferm. 2009;62(1):38-44.

17 Costa TF, Felli VEA. Exposição dos trabalhadores de enfermagem às cargas químicas em um hospital público universitário da cidade de São Paulo. Rev Latinoam Enferm. 2005;13(4):501-8.

18 Ministério do Trabalho e Emprego (BR), Superintendência Regional do Trabalho e Emprego do Rio Grande do Sul. Análises de acidentes do trabalho fatais no Rio Grande do Sul: a experiência da Seção de Segurança e Saúde do Trabalhador - SEGUR. Porto Alegre: Superintendência Regional do Trabalho e Emprego do Rio Grande do Sul. Seção de Segurança e Saúde do Trabalhador/SEGUR; 2008.

\author{
Endereço do autor / Dirección del autor / \\ Author's address \\ Leni de Lima Santana \\ Rua Maestro Carlos Frank, 2391, ap. 23, bl. 02 \\ 81750-400, Curitiba, PR \\ E-mail: lenisantana@hotmail.com
}

Recebido em: 18.07.2012

Aprovado em: 04.02.2013 